\title{
Sensitivity Improvement by Applying Lock-In Technique to Fluorescent Instrumentation for Cell-Based Odor Sensor
}

\author{
Totok Mujiono, Yuji Sukekawa, Takamichi Nakamoto, ${ }^{*}$ Hidefumi Mitsuno, ${ }^{1}$ \\ Maneerat Termtanasombat, ${ }^{1}$ Ryohei Kanzaki, ${ }^{1}$ and Nobuo Misawa ${ }^{2}$ \\ Tokyo Institute of Technology, 4259 Nagatsuta-cho, Midori-ku, Yokohama, Kanagawa 226-8503, Japan \\ ${ }^{1}$ Research Center for Advanced Science and Technology (RCAST), The University of Tokyo, \\ 4-6-1 Komaba, Meguro-ku, Tokyo 153-8904 Japan \\ ${ }^{2}$ Kanagawa Academy of Science and Technology, KSP East 303, \\ 3-2-1 Sakado, Takatsu-ku, Kawasaki, Kanagawa 213-0012, Japan
}

(Received April 13, 2016; accepted July 5, 2016)

Keywords: biosensor, odor sensor, olfactory receptor, fluorescence, lock-in technique

An odor-sensing system based on the insect olfactory receptor (OR) and fluorescent instrumentation has been developed. Cells expressing ORs were responsible for odor detection by monitoring the change in fluorescent light intensity emitted by the cells. Since fluorescent light from the cells is very weak, the measurement quality deteriorates if ambient light is present. A lockin measurement technique was incorporated to the system to overcome the problem of the ambient light together with the fluorescent image. The lock-in technique enables the detection of light synchronous with the modulated excitation light under the ambient light. This technique is useful for improving the signal-to-noise ratio. In this paper, sensitivity improvement by applying the lockin technique to fluorescent instrumentation for a cell-based odor sensor is discussed quantitatively. Experimental results show that the employment of the lock-in technique enhances the sensitivity by three orders of magnitude under the ambient light condition.

\section{Introduction}

An odor-sensing system is required in many fields such as food, environment, health, and perfumery. Some examples of its potential application are in the discrimination between good and contaminated drinking water and dairy products, detection and identification of bacteria and infectious diseases, and quality control in the food industry. An odor-sensing system typically has an array of sensors with partially overlapping specificities in the same manner as in humans or animals.(1) However, the sensitivity and selectivity of the odor-sensing system should be improved. Thus, a biosensor offers some advantages compared with traditional sensors based on metal oxides, polymer sensing films, and so forth.

In humans or animals, an olfactory receptor (OR) is essential for detecting odorants. ${ }^{(2)}$ A cell-based odor sensor is promising since it has high sensitivity, rapid response, and low cost. Moreover, it is easier to realize a biosensor with an insect OR than with a mammalian OR because of its simpler structure. Thus, we have adopted a biosensor with cells expressing insect ORs. ${ }^{(3)}$

*Corresponding author: e-mail: nakamoto.t.ab@m.titech.ac.jp http://dx.doi.org/10.18494/SAM.2017.1378 
We have used the fluorescent method to develop an OR-based odor biosensor. ${ }^{(4)}$ One of the main drawbacks in the fluorescence technique is the effect of ambient light whose intensity is much higher than that of fluorescent light from cells. ${ }^{(5)}$ To deal with a very weak signal buried under very strong unwanted noise, a lock-in measurement technique has been applied.(6) The purpose of this research is to develop a sensitive and portable fluorescent instrumentation system robust against ambient light using a lock-in measurement technique. The advantage of our sensor among optical biosensors is high robustness against ambient light.

Although the analyses of lock-in measurement techniques such as signal-to-noise ratio and dynamic range have already been reported, we experimentally studied the sensor sensitivity since the noise assumption is quite different from that found in the literature. ${ }^{(7-9)}$ The dominant noise in the fluorescent instrumentation system is ambient light, which is a DC signal with some fluctuation. In this paper, we discuss the sensitivity improvement by applying the lock-in technique to fluorescent instrumentation for cell-based odor sensors. This approach was taken since the ambient light is not always constant.

The experiments were performed under several ambient light conditions: dark (no ambient light), medium intensity, and high intensity. Although it was found in a previous study that the lock-in measurement was useful for decreasing the effect of ambient light, ${ }^{(6)}$ we have not known its performance. In this study, we aimed to find the detection limit using lock-in measurement in comparison with the conventional method under dark or ambient light conditions.

\section{Materials and Methods}

\subsection{Sensing mechanism and fluorescent instrumentation}

The cell-based sensing element used in this research is the insect OR expressed by Spodoptera frugiperda cells (Sf21) in which the fluorescent protein GCaMP is placed to monitor the intracellular calcium ion concentration. ${ }^{(3)}$ An illustration of the cell-based sensing element is shown in Fig. 1(a). A pair of ORs acts as a heteromeric ligand-gate ion channel, which has either an "open" or "closed" state. The odor-sensing mechanism starts when a pair of ORs binds to its associated odorant molecules to make the ion gate "open" and thus it allows calcium ions to flow into the cell. When a cell is illuminated by an excitation light $(488 \mathrm{~nm})$, the cells with more calcium ions inside emit brighter fluorescent light $(515 \mathrm{~nm})$.

A pair of ORs consists of OR and OR coreceptor (Orco). One of the ORs is OR56a from the fruit fly Drosophila melanogaster, which gives a specific response to geosmin, an earthy smell. There are 60 types of OR in the fruit fly genome. Furthermore, more than 100 types of insect ORs have been characterized and each of them responds to either one or several types of odorants. Since the intensity of fluorescent light produced by a single cell is very low and varies among the cells even if the OR type is the same, not a single cell but a group of cells is used as a sensor element.

On the basis of this mechanism, a fluorescent instrumentation system has been developed. ${ }^{(6)}$ The block diagram of the measurement system is shown in Fig. 1(b). It consists of a fluidic system, an optical system, and a lock-in measurement technique. The fluidic chamber is a microchamber made of polydimethylsiloxane (PDMS) material and the glass plate for the base to place the cells. This configuration enables both light excitation and detection of the fluorescent light from the bottom of the chamber. The cells are put on the chamber pool where odorant liquid flows. A reservoir, a solenoid valve, and a syringe pump were used to control the liquid flow. 


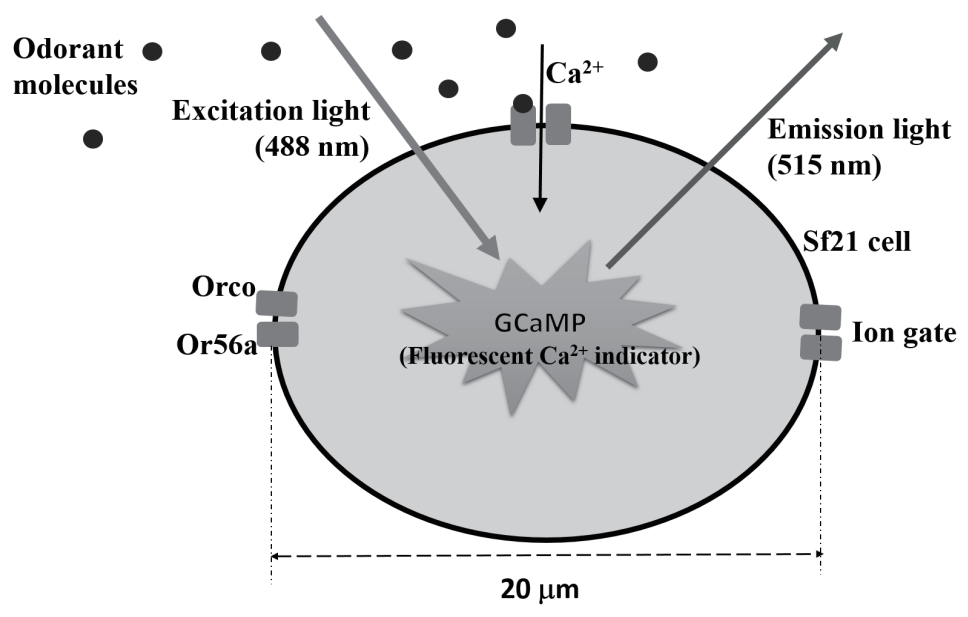

(a)

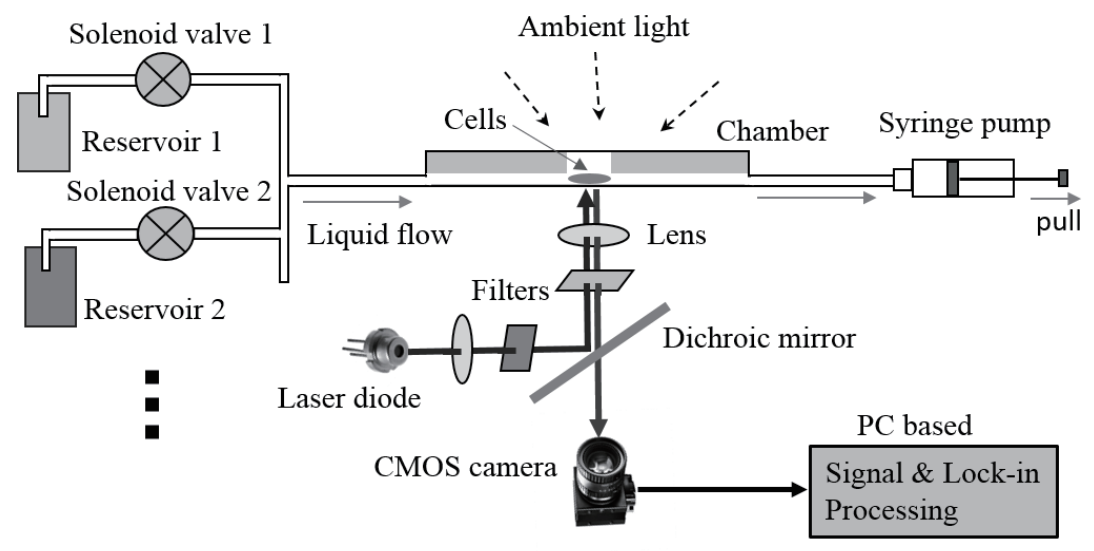

(b)

Fig. 1. Sensor principles and its instrumentation. (a) Sensing mechanism. (b) Schematic diagram of fluorescent instrumentation.

The optical system consists of lenses, a dichroic mirror, optical filters, a laser diode system, a complementary metal-oxide semiconductor (CMOS) camera, and lock-in processing. The laser diode system is used to provide the blue excitation light for the cell. It consists of a laser driver, a laser diode, and a dichroic mirror. A square-wave reference signal is applied by setting the laser driver in pulse mode. Lock-in processing consists of three blocks, which are a high-pass filter (HPF), a phase and frequency detector (PFD), and a low-pass filter (LPF). Currently, the lock-in processing is performed offline using Matlab.

The images of the cells as sensor element under various conditions are shown in Figs. 2(a)-2(c): dark condition [Fig. 2(a)], low intensity of ambient light [Fig. 2(b)], and high intensity of ambient light condition [Fig. 2(c)]. The small circles of around $20 \mu \mathrm{m}$ diameter shown in those images are the cells. It is difficult to observe the cell image intensity change under ambient light condition with high intensity. The sensitivity of the sensor deteriorates under ambient light condition, especially with high intensity. 


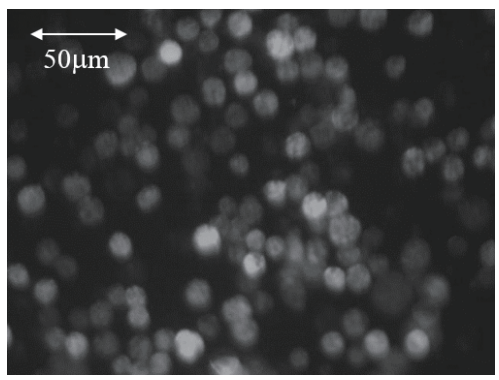

(a)

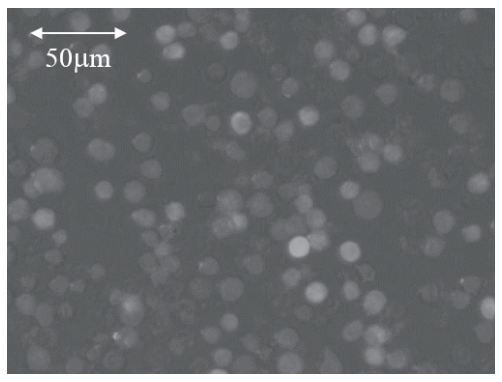

(b)

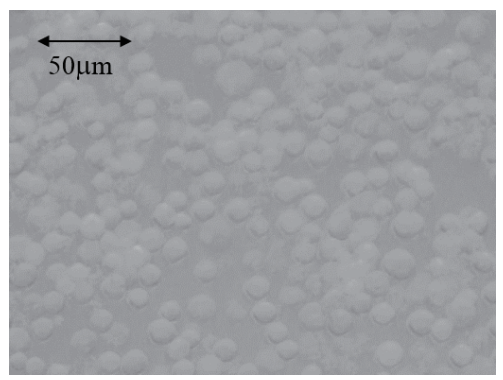

(c)

Fig. 2. Cell image capture by camera under various conditions. (a) Dark condition. (b) Low intensity of ambient light. (c) High intensity of ambient light.

Many cells are involved in the sensor, as shown in Figs. 2(a)-2(c). However, not all cells responded to the odorant and the responses differed from cell to cell. These characteristics are shown in Figs. 3(a)-3(b). Figure 3(a) shows the response curves of the Or56a cells to $10 \mu \mathrm{M}$ geosmin under dark condition. The upper line shows the sum of the intensities of all pixels in the image while the lower lines show the responses of five individual cells. The magnifications of the five individual cells' responses are shown in Fig. 3(b). It can be seen that the response varies in terms of intensity and transient behavior. Thus, it is essential to use a group of cells as a sensor to increase the response stability and decrease the variances among sensors. The maximum intensity changes are considered as the responses of the sensor to the odorant.

\subsection{Signal, noise, and lock-in measurement technique}

A CMOS camera captures not only the information signal $x(t)$ (fluorescent light) but also the unwanted output background $b(t)$, offset, and noise $n(t)$. This situation is shown in Fig. 4. Background light has intensity much higher than that of fluorescent light. Background signal mainly comes from ambient light such as sun and room lighting, thus, its intensity is not constant. Under the dark condition, the background light is minimal.

Offset is the intensity captured by a camera when there is no light. Its value is constant and depends on the camera setting, such as image type and exposure time. For example, the offset value in Fig. 4 is obtained from the CMOS camera under the condition of $450 \mathrm{~ms}$ exposure time. The noise $n(t)$ comes from several sources such as the thermal noise of the CMOS camera and laser diode, and cells moving during the experiment. The noise $n(t)$ normally has a smaller magnitude and a higher frequency than those of background and offset. The noise $n(t)$ affects the results of the measurement of a low concentration of odorant since the response is very low. The output $f(t)$ captured by the camera can be written as

$$
f(t)=x(t)+\text { offset }+n(t)+b(t) .
$$

\subsection{Lock-in measurement technique}

To deal with noises, a lock-in measurement technique has been added to the fluorescent instrumentation. The block diagram of the lock-in measurement technique adopted in this research 


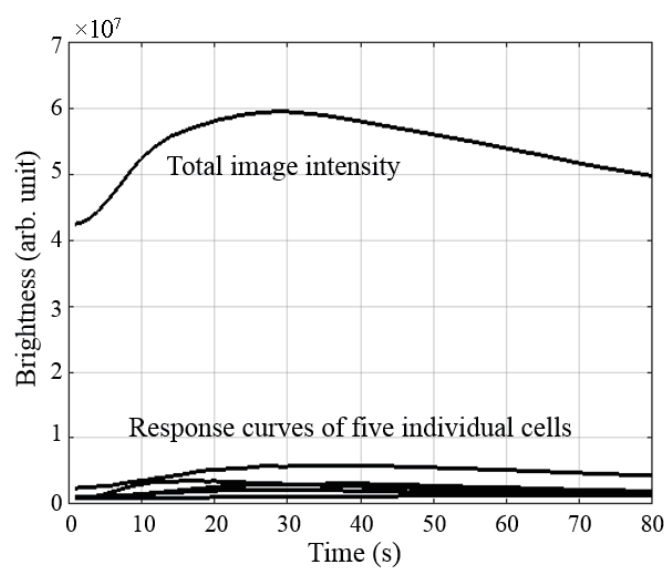

(a)

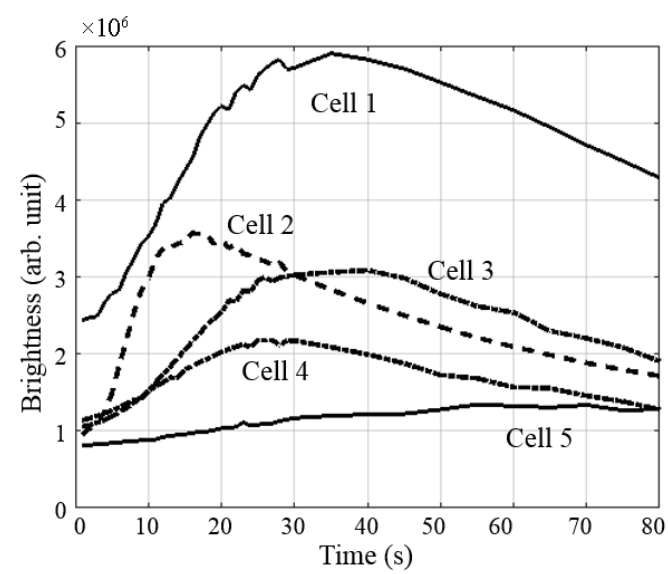

(b)

Fig. 3. Response curves of Or56a cells to $10 \mu \mathrm{M}$ geosmin odorant. (a) Total image intensity (upper) and five individual cells (lower). (b) Magnification of five individual response curves.

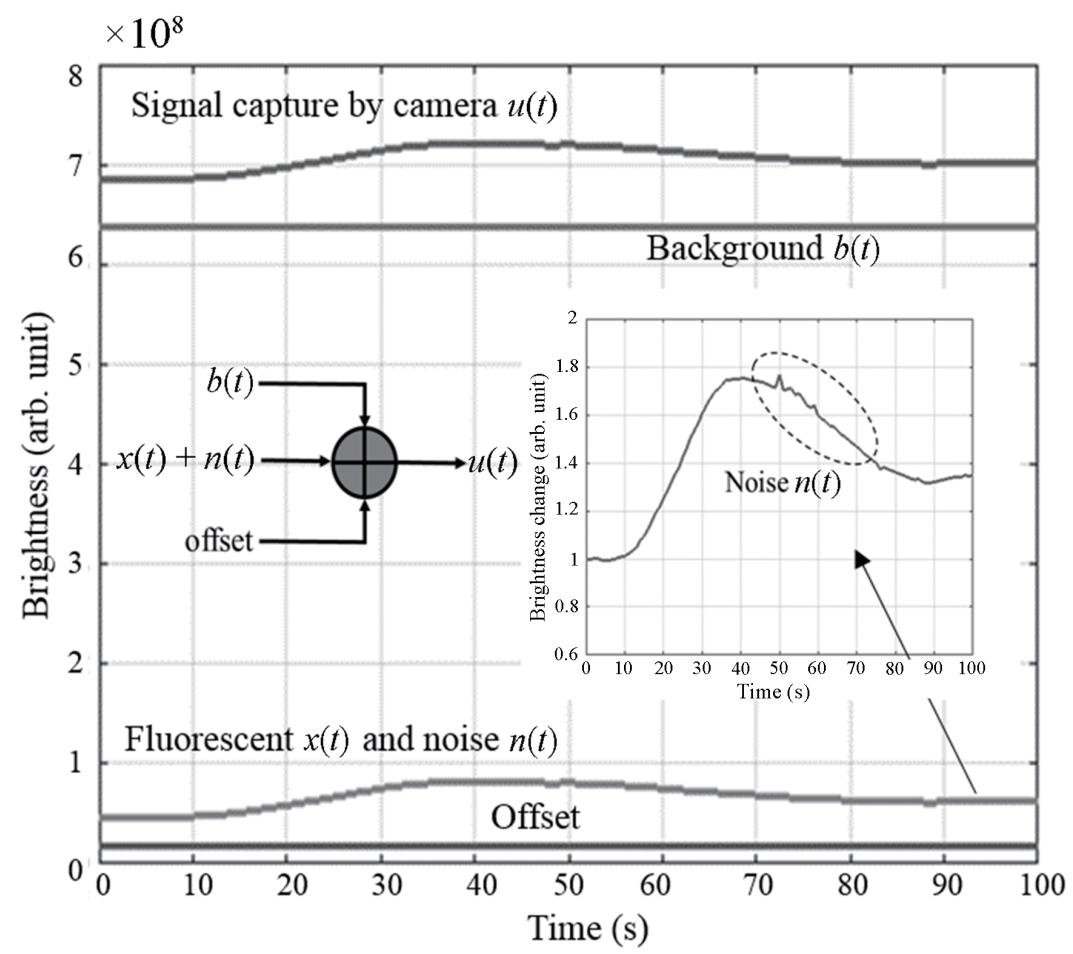

Fig. 4. Signal captured by the camera $[u(t)]$ under ambient light condition and its components including offset, fluorescent signal $x(t)$, noise $n(t)$, and background $b(t)$.

is shown in Fig. 5(a). The on-off modulation signal $m(t)$ is used to modulate the fluorescent signal $x(t)$ with noise $n(t)$. This modulated light mixed with background and offset is the signal captured by the CMOS camera $[u(t)]$, 


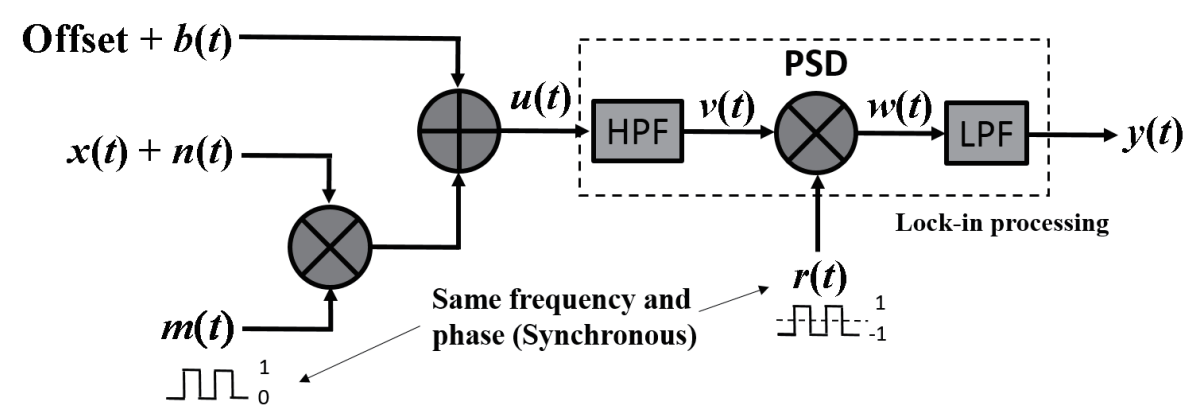

(a)

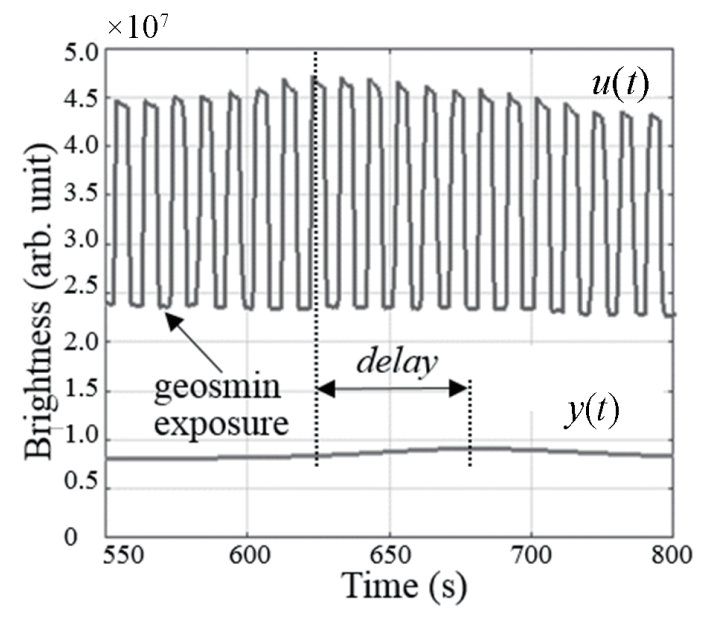

(b)

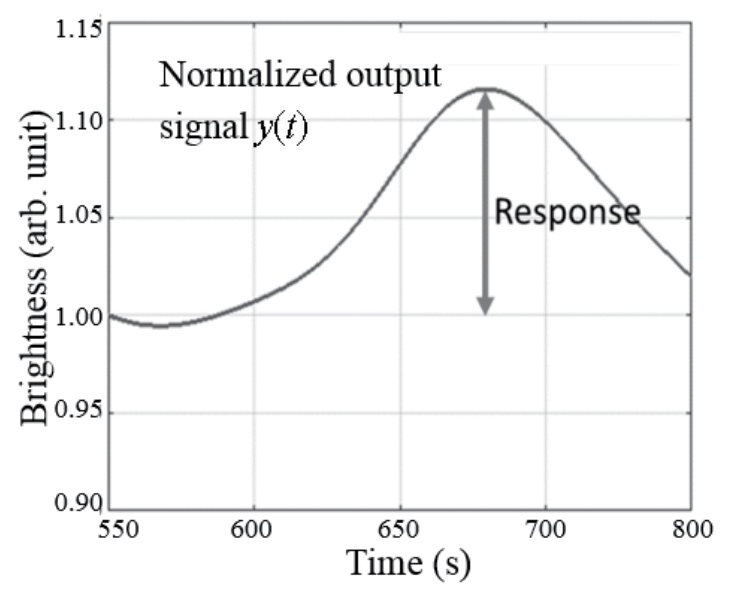

(c)

Fig. 5. Lock-in measurement technique in fluorescent instrumentation. (a) Block diagram of the lock-in technique. (b) Plot of responses of Or56a cells to $500 \mathrm{nM}$ geosmin. Signal captured by camera $[u(t)]$ and output signal after lock-in processing $[y(t)]$. (c) Normalized output signal and cells' response to $500 \mathrm{nM}$ geosmin $(11 \%)$.

$$
u(t)= \begin{cases}b(t)+\text { offset, } & \text { if } m(t)=0 \\ x(t)+n(t)+b(t)+\text { offset, } & \text { if } m(t)=1\end{cases}
$$

Modulation of the fluorescent signal was carried out by applying the pulse mode (PM) to the laser driver instead of the continuous mode $(\mathrm{CM})$ as in the normal condition. Lock-in processing was performed by filtering [high-pass filter (HPF) and low-pass filter (LPF)] and synchronous detection of the modulated signal using a phase-sensitive detector (PSD). HPF is used to remove the low-frequency contents of $u(t)$ [background $b(t)$ and offset] while LPF is used to remove the high-frequency contents [noise $n(t)$ ]. Thus, the remaining output signal $y(t)$ is the fluorescent signal $x(t)$.

In our case, lock-in processing (HPF, PSD with the reference signal $r(t)$, and LPF) was carried out offline under the platform of Matlab. The modulation signal $m(t)$ and the reference signal $r(t)$ were actually in the same phase since the modulation frequency was very low $(0.1 \mathrm{~Hz})$. The sampling frequency in our system was $1 \mathrm{~Hz}$, the cut-off frequency of HPF was set to $60 \mathrm{mHz}$, and the cut-off frequency of LPF was set to $30 \mathrm{mHz}$. Fifth-order Butterworth filters were used in this study. 
The processing of the intensity signal in the lock-in measurement technique is illustrated in Figs. 5(b) and 5(c). The upper plot in Fig. 5(b) shows the signal captured by the camera $[u(t)]$ for the cells expressing Or56a exposed to $500 \mathrm{nM}$ geosmin. The output signal $y(t)$ (lower curve) was obtained after the lock-in technique was applied. The delay was approximately $50 \mathrm{~s}$ due to the filter time constant. Figure 5(c) shows the normalization of the output signal by the brightness before the geosmin exposure. The response of the sensor to the odorant, defined as the maximum brightness change, was approximately $11 \%$ in this case.

\subsection{Experiment procedure}

\subsubsection{Experiment material}

Materials used in the experiment include cells, odorants, solvents, and Ringer's solution ( $\mathrm{pH}$ 7.2, and $140 \mathrm{nM} \mathrm{NaCl}, 5.6 \mathrm{nM} \mathrm{CaCl}_{2}, 11.26 \mathrm{nM} \mathrm{MgCl}_{2}, 19 \mathrm{nM}$ HEPES, and $9.4 \mathrm{nM}$ D-glucose). Two types of OR were prepared: Or56a and Or13a. Or56a responds to geosmin, the smell of mold produced by actinobacteria and can usually be found in drinking water. ${ }^{(10)}$ Or13a responds mainly to 1-octen-3-ol, the smell produced by most mycotoxigenic fungi and can be used as an indicator of fungal contamination in grain. ${ }^{(11)}$ The protocol of sf 21 cell lines was previously described. ${ }^{(3)}$ Two types of odorant, geosmin and 1-octen-3-ol, were prepared. Dimethyl sulfoxide, abbreviated as DMSO (99\% purity, Wako, Japan), was used to dissolve both geosmin (Wako, Japan) and 1-octen3-ol (98\% purity, Sigma-Aldrich, China) odorants into Ringer's solution. The concentration of DMSO in the odorant liquids was kept below $1 \% \mathrm{v} / \mathrm{v}$.

\subsubsection{Cell and odorant preparation}

To maintain the cells used for our experiment, they should be cultured every 3-4 days. The culturing was carried out by putting $1 \mathrm{~mL}$ of cells into fresh $4 \mathrm{~mL}$ of medium composed of Grace's insect medium (Gibco, Life Technologies, USA), fetal bovine serum (FBS), and antibiotics (10 $\mu \mathrm{g} /$ $\mathrm{mL}$ blatizidin, $300 \mu \mathrm{g} / \mathrm{mL}$ zeocyn, and $10 \mu \mathrm{g} / \mathrm{mL}$ gentamicin).

Before the experiment, Grace's insect medium (Gibco, LifeTechnologies, USA) was replaced with Ringer's solution. Then, the cells were taken from the base of the flask and were put into the microfluidic chamber through the hole on the top of the chamber. After approximately $20 \mathrm{~min}$, the cells were firmly attached to the base of the chamber and ready for odorant exposure. Or56a and Or13a were exposed to geosmin and 1-octen-3-ol, respectively. Ringer's solutions and odorant liquids with indicated concentrations were prepared in the reservoirs.

\subsubsection{Experiment on evaluation of sensor's performance with and without lock-in technique}

In the experiment, the odorant exposure was performed by flowing the odorant liquid with a specific concentration to the chambers for $60 \mathrm{~s}$ followed by Ringer's solution with the flow rate of $100 \mu \mathrm{L} / \mathrm{min}$. In this experiment, Or56a and Or13a cells were tested with geosmin and 1-octen-3ol, respectively. There were three ambient light conditions in the experiments: dark (no ambient light), low intensity (approximately $500 \mathrm{lux}$ ), and high intensity (approximately 1000 lux). For every ambient light condition, the experiments were performed both with and without the lock-in technique. Thus, for every type of cell, there were 6 experimental conditions: dark-without lock-in, 
dark-with lock-in, low intensity without lock-in, low intensity with lock-in, high intensity without lock-in, and high intensity with lock-in.

In the case of experiments without the lock-in technique applied, the excitation light (laser) was always ON. The laser driver circuit was run in continuous mode. Although there was no lock-in processing for the signal captured by the camera $[f(t)]$, the maximum responses after the normalization were obtained. In the case of experiments with lock-in measurement technique, the method was explained in the previous section.

The cell for experiments was prepared three or four days in advance and it was only used once in each experiment. At the current stage, although repeatable measurements are possible at the appropriate condition, we focused on the issue of lock-in measurement here. For every condition (dark, low ambient light, or high intensity of ambient light), the experiments were performed for many concentrations of odorant from $0 \mathrm{nM}$ to $100 \mu \mathrm{M}$. For every concentration under any condition, the experiments were performed five times to obtain valid data. These data were used for statistical analysis (t-test) to determine whether there was a significant difference between the condition with $0 \mathrm{nM}$ odorant concentration and that with a specified sample concentration.

\section{Experimental Results and Discussion}

\subsection{Response of Or56a cell line to geosmin}

The experiments were under three ambient light conditions both without and with the lockin technique applied. Some experimental results on Or56a cells with several concentrations of geosmin odorant are shown in Figs. 6 and 7. Figure 6 shows the typical response curves of the cells. Under high-intensity (1000 lux) ambient light without the lock-in technique applied, it was difficult to detect the geosmin concentration of $10 \mu \mathrm{M}$ since the response was very small $(4 \%$ or less) and was almost similar to the noise. While under a similar ambient light condition with the lock-in technique applied, the sensor system can be used to detect even $100 \mathrm{nM}$ concentration of the odorant. The sensitivity improvement was achieved by the detection of the signal synchronous with the reference signal while the ambient light not correlated to the reference signal was removed. When this signal was normalized, its magnitude obtained with the lock-in technique was higher than that without it.

Five experiments were carried out for any concentration chosen under any condition. Figure 7 shows the boxplot of the responses to characterize their variation. Each boxplot shows the minimum, lower quartile, median, upper quartile, and maximum responses. In every condition, the obtained responses from any concentration of geosmin were also compared with zero concentration using t-test. The p-value of less than 0.06 was used to indicate that a concentration under test has a significantly different response from that at zero concentration. The detection limit is the minimum concentration that has a significantly different response from that to zero concentration. The detection limit, indicated by the symbol *, for every condition is also shown in Fig. 7.

The detection limits under various conditions are shown in Fig. 8. Under the dark condition, the improvement obtained by applying the lock-in measurement technique is not significant (twice smaller), while at higher ambient light condition (500 lux), it becomes more significant (100 times smaller). The improvement becomes maximal at high intensity of ambient light (1000 lux), which was around 1000 times smaller. 


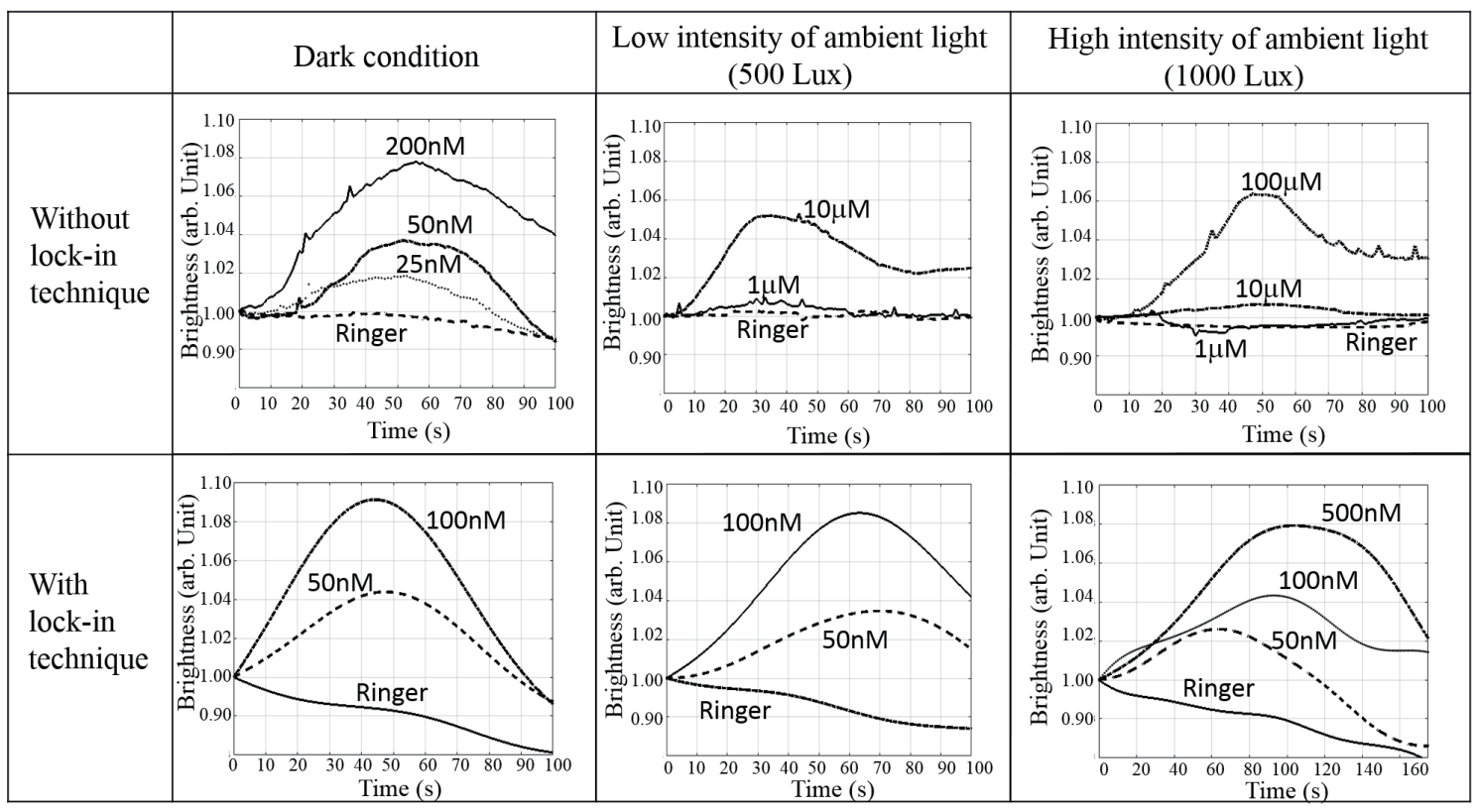

Fig. 6. Typical response curves of Or56a cell line to geosmin under various conditions.

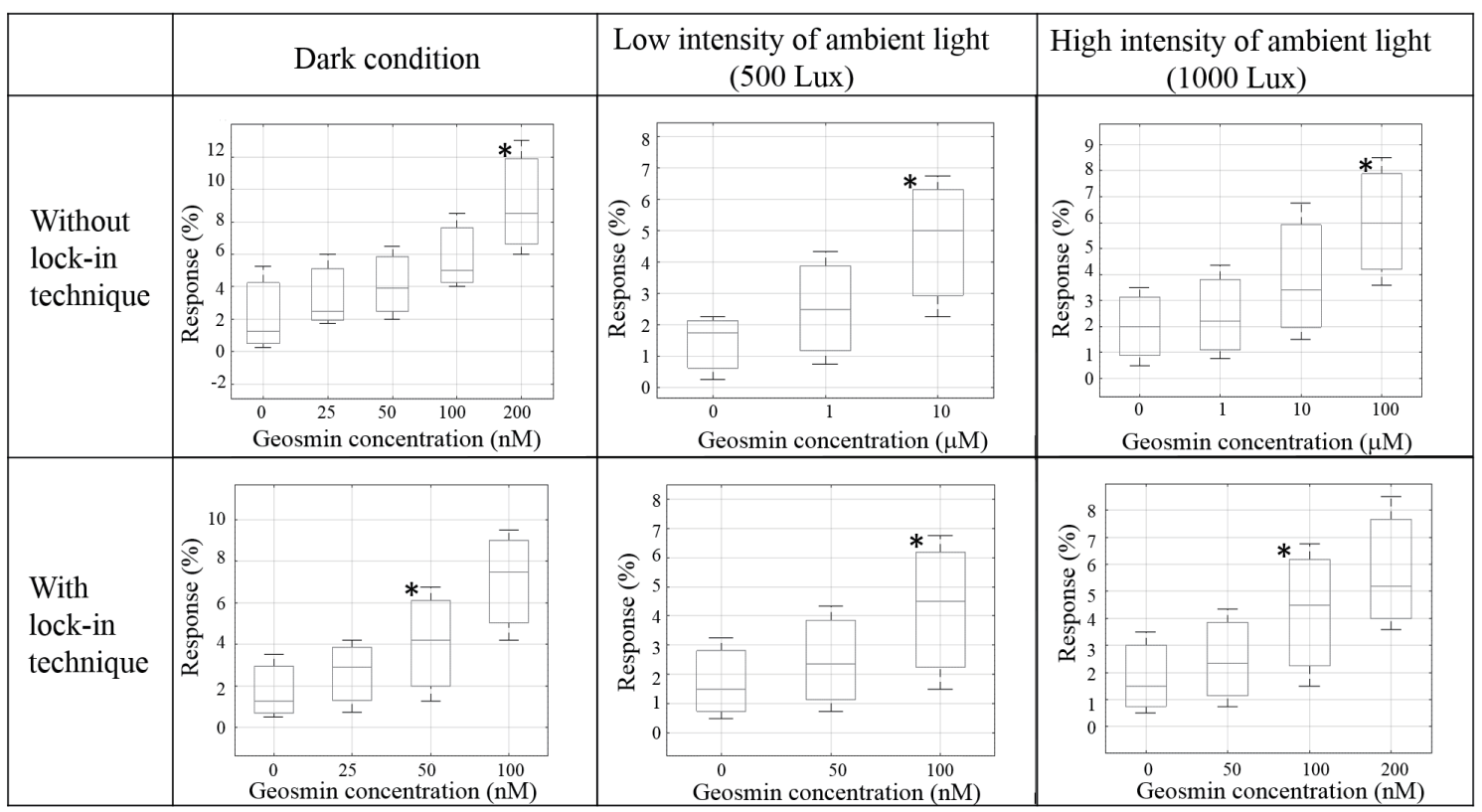

Fig. 7. Responses of Or56a cell line to geosmin under various conditions. The geosmin concentration with star $(*)$ is the detection limit for each condition.

\subsection{Response of Or13a cell lines to 1-octen-3-ol}

An experiment similar to that using Or56a above was applied to Or13a cell lines with 1-octen3-ol odorant. Figure 9 shows the typical response curves of the cells. In the experiment on the ambient light with high intensity (1000 lux) with the lock-in technique, it is possible to detect 50 


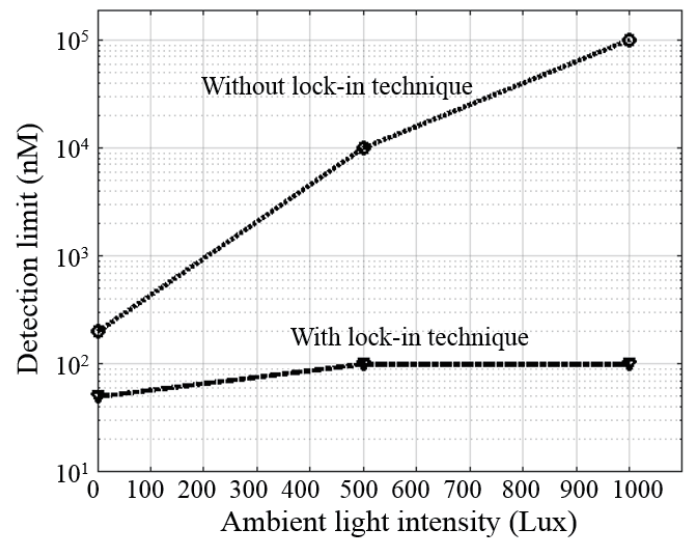

Fig. 8. Detection limit of Or56a cell lines to geosmin under three ambient light conditions with and without lockin technique applied.

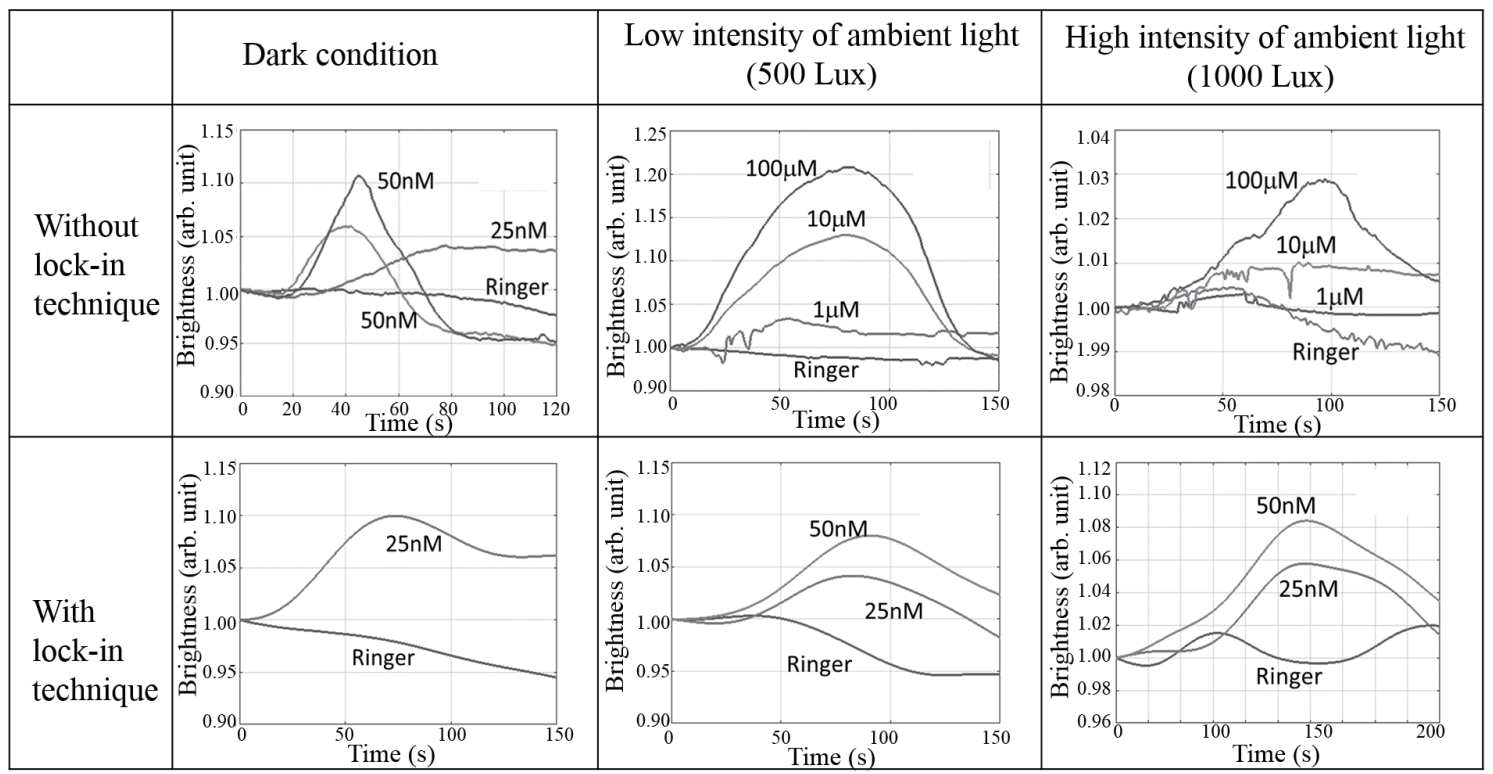

Fig. 9. Typical response curves of Or13a cell line to 1-octen-3-ol under various conditions.

nM 1-octen-3-ol odorant. Figure 10 shows the boxplot of the responses and their characteristics. The detection limit, indicated by the symbol *, for every condition is also given in the same figure. The detection limit under various ambient light conditions is summarized in Fig. 11. The detection limit improvement under the dark condition reached half of the concentration without the lock-in technique. The improvement becomes more remarkable under ambient light condition with its high intensity (2000 times). 


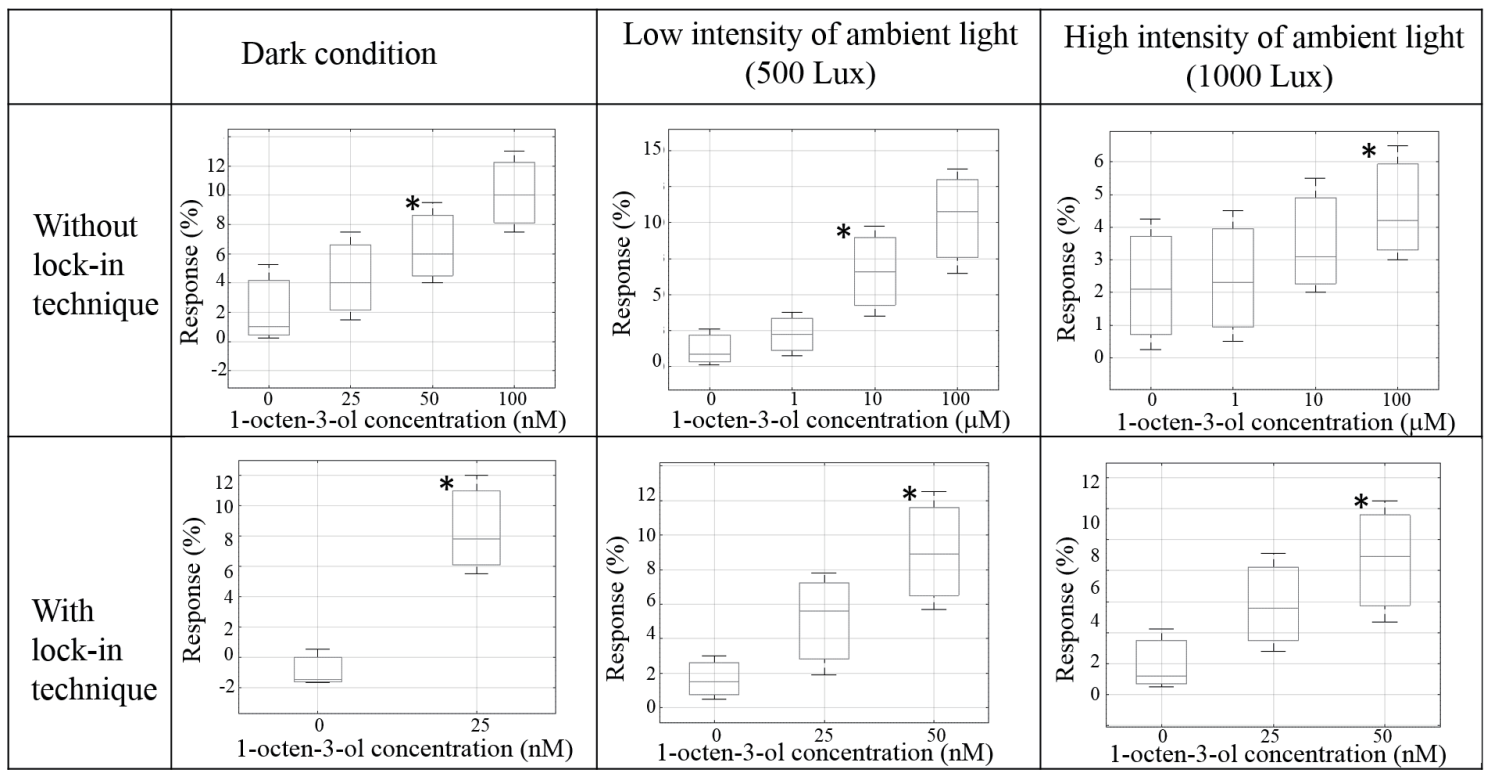

Fig. 10. Responses of Or13a cell line to 1-octen-3-ol under various conditions. The 1-octen-3-ol concentration with $\operatorname{star}(*)$ is the detection limit for each condition.

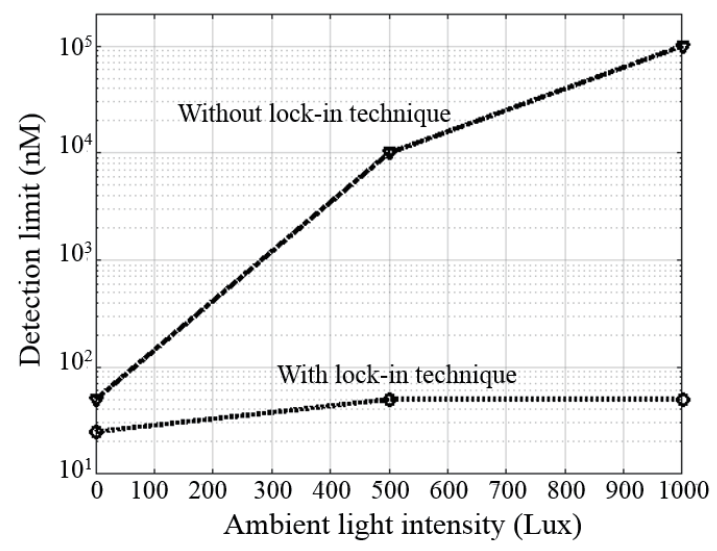

Fig. 11. Detection limit of Or13a cell lines to 1-octen-3-ol under three ambient light conditions with and without lock-in technique applied.

\section{Conclusions}

In this paper, the effect of the lock-in technique in our biosensor was thoroughly studied. It was found that the improvement of the detection limit reached approximately three orders of magnitude under the ambient light condition. The application of the lock-in technique has a significant impact on the measurement under ambient light condition. The detection limits of both cells were improved from $100 \mu \mathrm{M}$ without the lock-in technique to around 100 and $50 \mathrm{nM}$ with the lock-in technique for Or56a and Or13a, respectively. The dynamic range shows the region between the 
smallest and the largest detectable concentrations. The sensor works over the concentration range of 3-4 orders of magnitude. Although the lock-in measurement technique was useful, real-time processing has not been performed yet. We will soon extend it to real-time measurement.

\section{Acknowledgements}

This work was supported by the Japan Society for the Promotion of Science, Grant-in-Aid for Scientific Research (B) No. 25289120.

\section{References}

1 T. C. Pearce, S. S. Schiffman, H. Troy, and J. W. Gardner: Handbook of Machine Olfaction (Wiley-VCH Verlag, Weinheim, 2003).

2 L. Buck and R. Axel: Cell 65 (1991) 175.

3 H. Mitsuno, T. Sakurai, S. Namiki, H. Mitsuhashi, and R. Kanzaki: Biosens. Bioelectron. 65 (2015) 287.

4 T. Mujiono, Y. Sukekawa, T. Nakamoto, H. Mitsuno, R. Kanzaki, and N. Misawa: Proc. 2015 ASCC, Malaysia, 2015, 1.2-2.

5 J. C. Walters: J. Cell Bio. 185 (2009) 1135.

6 T. Mujiono, Y, Sukekawa, T. Nakamoto, H. Mitsuno, Y. Nakajima, R. Kanzaki, and N. Misawa: IEEJ Trans. Sens. Micromachine 136 (2016) 83.

7 M. L. Meade: Lock-in Amplifier: Principles and Application (Peter Peregrinus Ltd., London, 1983).

8 M. A. Rebolledo, J. C. Martin, M. Hotoleanu, M. Karasek, and E. Jaunart: Pure Appl. Opt. 5 (1996) 111.

9 A. De Marcellis, G. Ferri, A. D'Amico, C. Di Natale, and E. Martinelli: IEEE Sensor J. 12 (2012) 1377.

10 M. C. Stensmyr, H. K. M. Dweck, A. Farhan, I. Ibba, A. Strutz, L. Mukunda, J. Linz, V. Grabe, K. Steck, S. Lavista-Llanos, D. Wicher, S. Sachse, M. Knaden, P. G. Becher, Y. Seki, and B. S. Hansson: Cell 151 (2012) 1345.

11 J. Schnurer, J. Olsson, and T. Borjesson: Fungal Gen. Bio. 27 (1999) 209. 